\title{
Flujo documental entre áreas administrativas de una entidad bancaria: una aproximación desde el análisis de redes sociales (ARS)
}

\author{
Juan Bernardo Montoya Mogollón \\ Telma Campanha de Carvalho Madio \\ Universidade Estadual Paulista Júlio de Mesquita Filho - UNESP, Brasil
}

\section{ARTICLE}

\begin{abstract}
Resumen
El presente trabajo pretende como objeto de estudio, analizar doce departamentos administrativos de una entidad bancaria y sus relaciones en términos documentales (flujos), abordados desde la unidad de investigación del Análisis de Redes Sociales (ARS). El problema esencial de este estudio, se enfoca en determinar la manera en la que se relacionan las áreas descritas, destacando sus principales fortalezas y/o debilidades tanto al interior de cada una de ellas, como en sus relaciones con las demás en la organización. Por último, estos análisis pretende brindar la posibilidad de entender cómo se relacionan los actores dentro de una organización, para proyectar posibles soluciones en términos de relaciones y diseminación documental, una actividad que sigue teniendo visibles dificultades en la actualidad.
\end{abstract}

Palabras clave

Análisis de redes sociales ; Organizaciones ; Capital socia ; Diseminación de la información ; Gestión documental, Centralidad de grado ; Centralidad de cercanía ; Centralidad de intermediación

Documentary flow between administrative areas of a bank: an approach from the analysis of networks (SNA)

\begin{abstract}
This paper analyses twelve administrative departments in a bank and their relationships in documentaries terms (flows). The study was developed by a research unit applying Social Network Analysis (SNA). The objetive of this research was identify the relationship ways between these areas, highlighting their strengths and / or weaknesses, and their relations with others areas in the organization. Finally, this analysis tries to offer the opportunity to understand how actors relate within an organization, to project possible solutions in terms of relationships and documentary dissemination, an activity with visible difficulties still today.
\end{abstract}

Keywords

Social network analyses ; Companies ; Social capital ; Information dissemination ; Records management ; Degree centrality ; Closeness centrality ; Betweenness centrality 


\section{Introducción}

Sería interesante conocer una fórmula generalizada en la que las diferentes entidades organizacionales lograrán afrontar sus diversos y cambiantes problemas que surgen en el día a día durante sus operaciones laborales, especialmente en este tiempo de cambios constantes, donde la tecnología -ahora más que nunca- se encuentra en su "edad dorada" de desarrollo. Afortunada, o desafortunadamente, esas fórmulas no existen, pues cada organización determina sus propias especificidades y dinámicas de producción dependiendo de los factores que se estén comercializando.

Ahora bien, uno de los principales pilares para el correcto desempeño de cualquier empresa, se centra en su capital social y en las relaciones informacionales que se establecen al realizarse esas diversas interacciones. Estas, se configuran dentro de una estructura organizacional, gracias principalmente al protagonismo de los individuos al momento de desempeñar sus actividades dentro de un ambiente laboral.

Por tanto, es conveniente señalar la importancia del concepto de "capital social" estudiado, desarrollado y ampliado desde diferentes áreas investigativas, entre las que se destaca principalmente la sociología, donde el autor Julio César Rodríguez da una definición puntual al decir que:

No obstante, en un intento de facilitar la comprensión de este abstracto concepto, algunos investigadores como Pierre Bourdieu y Loïc J. D. Wacquant (1992), Ronald S. Burt (1992 y 2000), James Samuel Coleman (1990) y Robert Putman (1993) han asimilado el concepto de capital social a las ventajas derivadas de las mejores redes de conexiones que poseen algunas personas o algunos grupos de personas, lo cual sienta las bases para estudios sobre los vínculos entre actores sociales. Para tal entretejido entre las redes sociales y su análisis, dichos autores recurren a recursos literarios como la metáfora (social capital metaphor o metáfora del capital social) para transmitirlo y estudiarlo" (2013, p.105).

De esta manera, en este trabajo se tendrá en consideración este concepto para analizar específicamente la función del grupo social estudiado el cual pertenece a un ámbito laboral financiero. Sin embargo, para abordar el concepto con mayor profundidad y detalle, se sugiere analizar los textos que amplían el uso de este concepto, entre los que se encuentran además de los citados anteriormente, los de Janine Nahapiet y Sumantra Ghoshal (1998), Regina Marteleto y Antonio Silva (2004).

Desde hace algunos años, era impensado que el flujo de información establecido entre los individuos al interior de una empresa, fuera relevante para dar soluciones alternativas a las diversas dificultades que se presentan en el ámbito laboral, sin embargo, con el surgimiento de unidades de análisis investigativas para observar el comportamiento documental entre las que se encuentran el Análisis de Redes Sociales (ARS), se empezó a configurar una perspectiva para determinar esas relaciones, principalmente con la recolección de información específica que ayudó a observar el grado de cercanía o lejanía de un actor respecto al otro en términos documentales, dentro de un espacio social determinado.

De esta manera, el objeto central del presente trabajo se centra en aplicar la unidad de ARS para determinar el flujo documental que se distribuye al interior de una entidad financiera entre diferentes actores que pertenecen a 12 áreas administrativas. Esto, con el objetivo de ofrecer una propuesta alternativa a los problemas que surgen diariamente en el "flujo" documental de diferentes departamentos organizacionales, los cuales muchas veces no llegan a ser efectivos para la continuidad del negocio, término de vital importancia para el éxito organizacional.

La metodología igualmente aplicada en la presente investigación, fue tanto cuantitativa, -ya que se analizó un universo de actores que se comunicaron documentalmente dentro de la organización financiera- y cualitativa, ya que estos actores tuvieron una función dinámica al interior de la organización, los cuales construyeron un conocimiento tácito y explicito constantemente.

Así, esta herramienta de investigación solo fue aplicada a 12 áreas administrativas, ya que el número total era de 22 departamentos, y el número de empleados en el momento de la investigación superaron los 200. La muestra anterior se realizó con las áreas y colaboradores de "mayor influencia" en la organización al momento de construir la red en el software Ucinet, y se tuvo como fuente principal para este trabajo, una herramienta utilizada en el momento de levantar la información llamada "formato trámite", donde se plasmó la información ofrecida por cada uno de los trabajadores durante una serie de entrevistas abiertas. 
Con lo escrito anteriormente, este trabajo está estructurado en cuatro partes que se van a constituir en una importante ayuda para abordar el concepto de ARS de la siguiente forma: en un primer momento se abordará este concepto; su desarrollo y evolución histórica hasta nuestros días; los principales conceptos que se recogen en esta área y la manera en la que son aplicados en actuales investigaciones.

Posteriormente, se analizará el aporte del ARS al ámbito organizacional. Cuáles han sido sus principales contribuciones y qué investigaciones se han realizado en la actualidad, con el fin de observar la implicación y efectividad de esta perspectiva de investigación para el dominio organizacional.

Finalmente, se establecerá un estudio de caso de una entidad financiera, en la cual, con base en informaciones recogidas fruto de un proyecto de Gestión Documental que se realizó en el año 2013, se pudo llevar a cabo el Análisis de Redes Sociales, donde se determinaron las relaciones establecidas en diferentes áreas: cuáles áreas fueron las más centrales al momento de analizar la diseminación de la información; cuáles eran las más relegadas en este proceso de flujo de información y cuáles fueron los actores principales de la red organizacional en términos de centralidad, cercanía e intermediación. Por último, se darán las conclusiones del trabajo.

\section{Análisis de redes sociales, una breve construcción teórica hasta nuestros días}

Una red social es una construcción que se inicia entre mínimo dos individuos al momento de comunicarse y establecer un vínculo o relación. Contrario a lo que se piensa en la actualidad, el surgimiento de este concepto no es reciente, ya que la creación de grupos y redes sociales es un fenómeno que históricamente ha existido, y su análisis se empezó a estudiar desde principios del siglo XX, especialmente desde la década de los años 30 , donde se analizó un grupo de actores y las redes que establecían como parte de actividades terapéuticas (Rodríguez, 2013).

Posteriormente, en los años de la década de los 50's se comenzaron a hacer estudios de la teoría de grafos desde la antropología; en los años 60's se llevaron a cabo otros estudios desde la sociología; y en los años 70's desde la computación, se comenzó estructurar lo que sería hoy en día el ARS. Estas disciplinas generaron esa unidad de investigación transversal, que más adelante se aplicó a otras disciplinas como lo fueron las ciencias políticas, ciencias de la salud, la física, la economía e incluso la historia (Rodríguez, 2013).

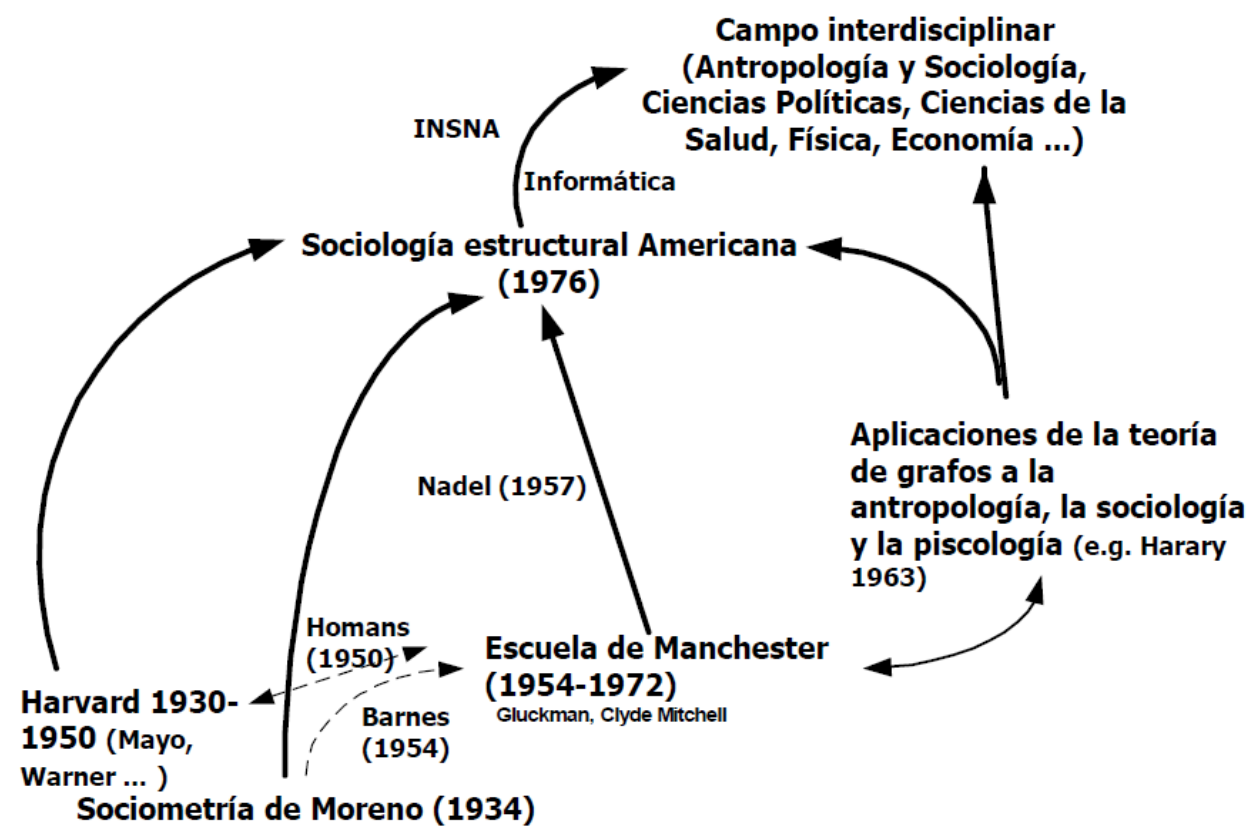


De esta manera, para contextualizar lo descrito, en la gráfica 1 se puede observar la evolución del ARS y las disciplinas originarias o fundacionales que utilizaron este concepto como una ayuda de aplicación para sus problemas teóricos.

Así, un aspecto a considerar en la figura 1, es la importancia de la sociología como una disciplina central que ha contribuido de manera constante al proceso de consolidación del ARS, debido a que la esencia de esta disciplina se basa en el estudio del individuo y la forma en la que él se comporta y se relaciona en sociedad.

Por otro lado, la aplicación de esta unidad de investigación está empezando a fortalecerse en los últimos años y esto se observa en investigaciones materializadas en revistas científicas que se enfocan en estudios de ARS, entre las que se encuentran principalmente la "Social Science \& Medicine, Health Policy, International Journal of Nursing Studies, Journal of Nursing Management, Chronic Illness, Journal of Health and Social Behavior y la Revista Hispana para el Análisis de Redes Sociales (Redes) de la Universidad Autónoma de Barcelona y el LRPC de la Universidad de Sevilla", entre otras (Marqués et al, 2013).

Por tanto, como lo explican los autores Renato Matheus y Antonio Silva, el ARS tienen un amplio campo de aplicación, especialmente en las redes establecidas en "personas y empresas, analizadas como unidades individuales, o como unidades sociales colectivas, como, por ejemplo departamentos dentro de una organización, agencias de servicio público en una ciudad, estados-naciones de un continente o del mundo (Wasserman; Faust; 2006, original en portugués)

Otro punto importante para señalar en el enfoque de ARS, se centra en el hecho de que en este campo se estudia principalmente las relaciones (Ties) de los actores (Nodes) dentro de una red y en un segundo grado, los atributos o características que estos actores poseen:

\begin{abstract}
En el Análisis de Redes Sociales, los atributos observados a partir de los actores sociales (como raza o grupo étnico de las personas, o el tamaño o productividad de cuerpos colectivos, tales como empresas o estados-naciones) son comprendidos en términos de patrones o estructuras de relaciones entre las unidades. Las ligaciones relacionales entre actores son el foco primario y los atributos de los actores son secundarios (Wasserman; Faust; Matheus, Silva, 2006, original en portugués).
\end{abstract}

Ahora, dentro de los problemas teóricos inherentes a las Ciencias de la Información (Cl), esta perspectiva de ARS, está empezando a constituirse en una fuente interesante para dar posibles alternativas de solución y para desarrollar un campo de investigación que está creciendo cada vez más en la actualidad. Su inclusión dentro del dominio de las $\mathrm{Cl}$, se debió igualmente a su relación con los estudios bibliométricos. Un ejemplo de lo anterior, se puede observar en el estudio realizado por Otte y Rousseau (2002), donde establecieron desde la bibliometría, la producción de autores y utilizaron el ARS para estudiar esas relaciones de co-autoría, y gracias a las redes que construyeron de los autores determinados, se permitió analizar por ejemplo, las medidas de centralidad para observar los autores principales y sus relaciones con los demás (Matheus, Silva, 2006).

Igualmente, el ARS ha venido siendo utilizado en otras áreas dentro de las $\mathrm{Cl}$ y aunque no es aceptado propiamente como una herramienta conceptual de esta área de conocimiento, su relación con la estructuración y recuperación de información (RI) ha sido también importante; especialmente cuando los individuos comienzan a generar información y la comparten con sus semejantes, en ese momento el ARS es importante para determinar por ejemplo, ese flujo de información (directa o no) que se establece desde los sistemas de información (Matheus, Silva, 2006).

Finalmente, otro aspecto a considerar dentro del ARS es su evolución constante. Como se escribía al comienzo de esta sección, los conceptos adoptados especialmente por la sociología, la antropología y la psicología social, fueron determinantes para analizar las redes sociales que se establecen dentro de cualquier campo. En este sentido, se observan trabajos realizados en investigaciones relacionadas con "el impacto de la urbanización en el bienestar del individuo, los sistemas de economía y política, el apoyo social y el campo organizativo. Con respecto a este último, se ha estudiado la relación de las redes en cuanto al rendimiento, la creatividad, las transferencia de innovación, etcétera" (Marqués et al, 2013).

Ahora, otros términos incluidos en este campo, son los de la teoría de grafos y la formalización matemática, conceptos que han sido establecidos desde la tecnología (Marqués et al, 2013), por lo que su desarrollo se materializó en los diferentes softwares que se disponen en en la actualidad, de los cuales se pueden destacar: 
Gephi, Pajek, Ucinet, ORA, entre muchos más. Por lo tanto, para el objetivo de este trabajo, se va a utilizar el software Ucinet, el cual tiene incorporado una herramienta para graficar las redes llamado Netdraw.

Por último, a continuación se van a describir los principales conceptos que se establecen dentro de la perspectiva de ARS, los cuales, algunos de ellos van a ser utilizados en la parte aplicada de este trabajo.

\section{Conceptos de mayor desarrollo en el Análisis de Redes Sociales}

Utilizando un lenguaje práctico, esta sección se propone analizar los principales conceptos desarrollados dentro del ARS, los cuales han fortalecido y clarificado las investigaciones realizadas en este ámbito, convirtiéndolo igualmente en una perspectiva o área con un lenguaje autónomo. No se pretende elaborar un manual donde se listen todos los conceptos de ARS, sino establecer un mejor entendimiento del mismo para abordar el estudio de campo que se realizó.

Inicialmente, se hablará del concepto de red social, el cual es definido como la relación social que se establece entre dos o más individuos como se explicaba anteriormente, y la densidad de red, la cual se centra en analizar cuántos nodos están conectados de manera global. Esta relación también está definida por el tipo de grado dentro de la estructura social, es decir, puede ser una relación entrante (in-degree), cuando un nodo tiene un grado de entrada; o puede ser una relación saliente (out-degree), cuando un nodo tiene un grado de salida a otro nodo; o, puede ser de las dos formas (in-degree/out-degree), en caso de que los dos nodos estén conectados mutuamente. Este tipo de relaciones se expresan dentro del ARS con puntos (o figuras geométricas) y líneas (la dirección de las flechas indica el grado de amistad) como se muestra a continuación:

Figura 2: Red social en grafos

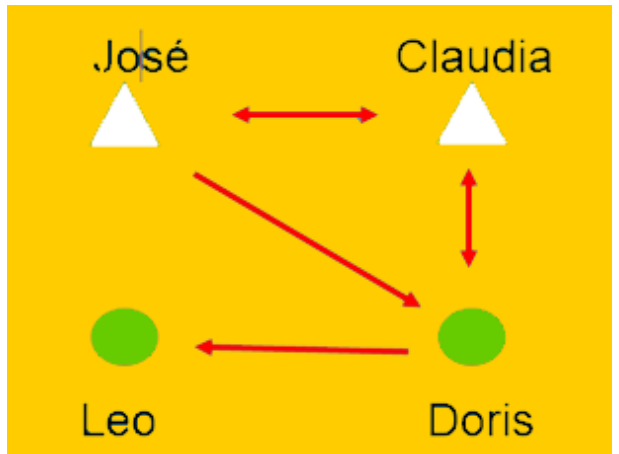

Fuente: (Hilbert, 2013)

Figura 3: Red social en matriz de datos

\begin{tabular}{|c|c|c|c|c|c|}
\hline & José & Claudia & Doris & Leo & Suma \\
\hline José & 0 & 1 & 1 & 0 & 2 \\
\hline Claudia & 1 & 0 & 1 & 0 & 2 \\
\hline Doris & 0 & 1 & 0 & 1 & 2 \\
\hline Leo & 0 & 0 & 0 & 0 & 0 \\
\hline Suma & 1 & 2 & 2 & 1 & 6 \\
\hline
\end{tabular}


De esta manera, en la gráfica 2 se muestra una relación de amistad de cuatro amigos: José, Claudia, Doris y Leo. José está conectado a Claudia y Claudia también está conectada a José; a su vez José está conectado a Doris, pero ella no está conectado con José; finalmente José no conoce a Leo pero tiene una amiga en común que sí conoce a Leo. Y así, se puede continuar extrayendo mayor información según sea la necesidad del investigador.

Otra forma de ver este tipo de relaciones es por medio de una matriz donde se tiene la información expresada de manera simétrica como se observa en la gráfica 3. Finalmente, esta información debe estar acompañada por otro concepto llamado atributo(s), con el objetivo de que la red tenga información de mayor complejidad y sea "más parecida" en el campo real. Un atributo de la red de amistad analizada en la matriz se simplifica en que hay dos mujeres y dos hombres y su grado de amistad. Pueden incluirse igualmente atributos adicionales como, niveles de ingresos económicos, edades de los actores (nodos), lugares de residencia, entre otros.

Para cuantificar el grado de conexión entre los actores, el ARS estableció numéricamente su relación: 1, cuando se está conectado, y 0 cuando no se está conectado. De esta manera, se puede observar que la red de amistad global de la gráfica 2 y 3, tiene 4 enlaces y 6 grados, es decir cuatro líneas y 6 flechas. Así, a manera de ejemplo, se puede observar que Claudia es el nodo que está más conectado con sus amigos con 4 grados de conexión ( 2 grados entrantes y 2 grados salientes).

Continuando con la terminología, se establece dentro del ARS cuando se "pasea" la red, el concepto de caminos (walks), el cual es definido como el paso a través de todos los nodos de la red a través de enlaces. Otro concepto, es la ruta (path), significa que se pasa por todos los nodos, pero nunca se pasa el mismo nodo más de una vez. Un ciclo, es un concepto en el que se termina un camino en el mismo nodo donde se comenzó. La geodésica, es el camino más corto entre dos nodos. El diámetro de la red, es la geodésica mayor entre dos nodos. Y el promedio de la ruta, la cual es el promedio de la geodésica entre los nodos.

Ahora, para adentrarnos en la red y analizar su especificidad y nivel de detalle, se va a abordar el término de la Centralidad de la red y los conceptos que de él se desprenden: Grado de Centralidad (o grado) (Centrality Degree), el cual hace referencia a aquel nodo que está mejor conectado dentro de la red, tanto de grados entrantes como de grados salientes; en este sentido se establece la importancia del autor cuando este tiene la mayoría de vínculos dentro de la red establecida. Tomando como ejemplo las gráficas 2 y 3 , el nodo que tiene un alto grado de centralidad dentro de la red es Claudia ya que posee 4 grados de conexión.

Centralidad de Cercanía (Closeness Centrality), es la medida que indica que tan cerca está un nodo respecto a los demás nodos, es decir, aquel que está a menos pasos (distancia geodésica) de los otros actores. Para esta medida de centralidad se va a tomar otro ejemplo, ya que se debe generar con nodos que estén representados de forma bidireccional como se muestra en la figura 4, donde se calcula el número de pasos del actor Silverio Vásquez al actor Rafael Amezcua, el cual como se observa en la imagen, son tres:

Figura 4: Centralidad de Cercanía

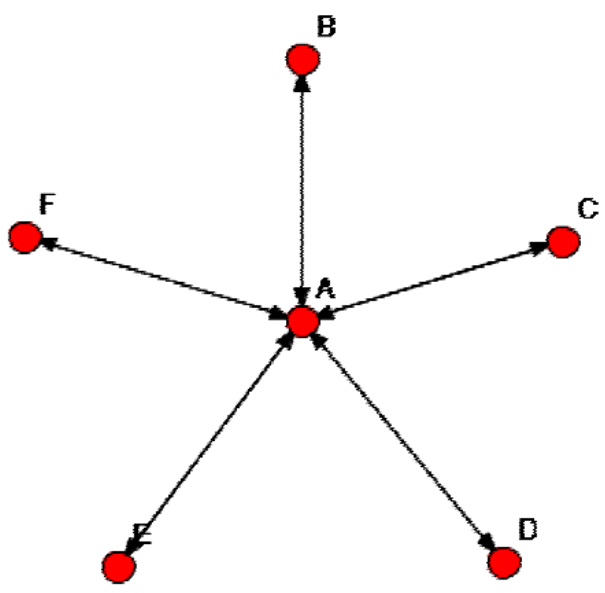

Fuente: (Velázquez, Aguilar, 2005) 
Finalmente, se analiza el término Centralidad de Intermediación (Betweenness Centrality), en el que se cuenta cuántas veces un nodo está en el camino (ruta geodésica) que conecta los otros nodos. Es decir, es una medida para calcular la centralidad o importancia de un nodo intermediador, "esta se enfoca en el "control de la comunicación", y se interpreta como la capacidad que tiene un nodo o actor para intermediar las comunicaciones entre pares de nodos". A estos nodos de intermediación también se les llama actores puente (Velázquez, Aguilar, 2005).

En la figura 5, se muestra el nodo A como el nodo con mayor grado de centralidad de intermediación, ya que hace de puente para los otros nodos de la red se puedan conectar entre sí:

Figura 5: Centralidad de Intermediación

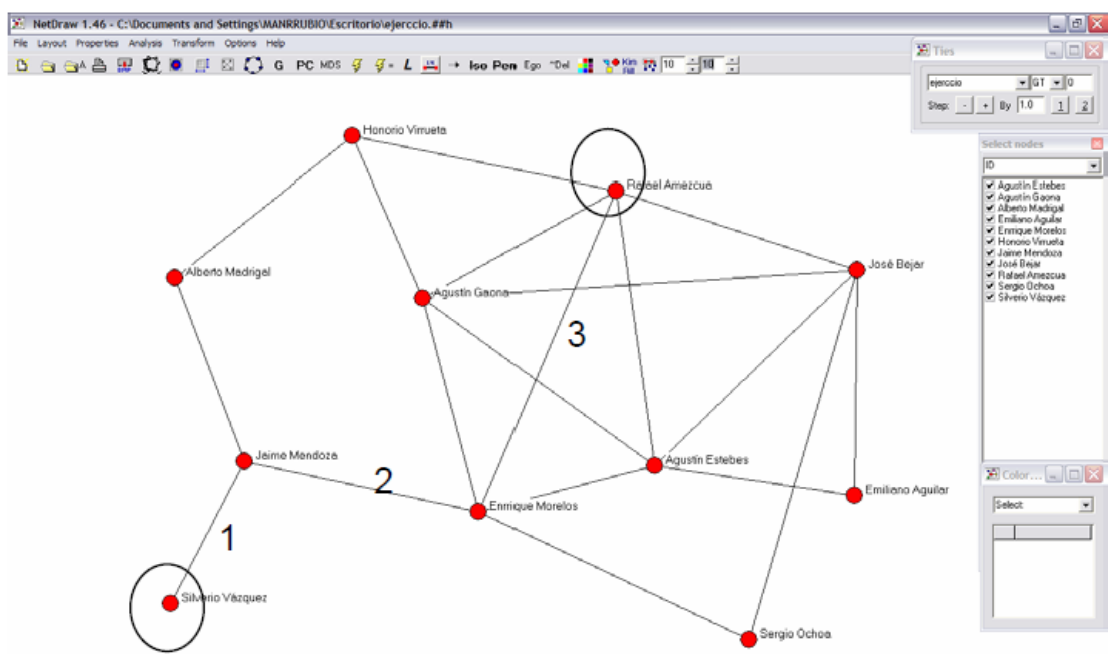

Fuente: (Velázquez, Aguilar, 2005)

\section{Aportes desde el Análisis de Redes Sociales al ámbito organizacional}

El concepto de Análisis de Redes Sociales continúa su ascenso y cada vez más son las empresas que utilizan este tipo de proyectos para "reorganizar" su estructura organizacional. Este tipo de ayudas son relevantes ya que los tradicionales organigramas, solo muestran una parte superficial e inamovible de cualquier entidad, al contrario del ARS que van directamente al comportamiento y al flujo de información de los actores que componen un área o un departamento, generando toma de decisiones que pueda beneficiar la continuidad del negocio.

De esta manera, se pueden observar trabajos relacionados con ARS en el ámbito organizacional, como por ejemplo el de Alejandro García (2000), el cual establece las relaciones sociales que establecen los empresarios de medianas y pequeñas empresas. El autor utiliza el concepto de "clusters" empresariales, precisamente para hablar de los conjuntos de empresarios que establecen sus redes sociales en una región de México.

Así mismo, en el trabajo de los autores Pilar Marqués, Marta González, Yolanda Agra, Jorge Vega, Arrate Pinto y Enedina Quiroga (2013), se analizan las relaciones sociales en una organización sanitaria, donde se estableció la fuerte relación que sostienen los médicos y los enfermeros, a diferencia de la poca conectividad con los auxiliares de esa entidad. $Y$ aunque los autores no encontraron una utilidad efectiva al análisis realizado, Io cierto es que en la red social elaborada, se observa que se debe aunar esfuerzos para que esa red establecida pueda ser más densa, con el objetivo de atender a los pacientes de una manera más efectiva.

Continuando, se analiza el estudio de Joaquim Fialho (2015), el cual tiene un campo de trabajo similar al propuesto en este artículo, sin embargo, el autor se centra en describir las "estrategias de cooperación interorganizacional entre trabajadores de una red de agencias bancarias en la región de Alentejo", ubicada en el centro-sur de Portugal. 
Igualmente, se encontró en esta búsqueda bibliográfica, un estudio realizado por el autor Álvaro Pina (2012), en el que se realiza un interesante análisis de actividades económicas de redes inter-organizacionales.

Finalmente, existen otros trabajos resumidos por los autores Renato Matheus y Antonio Silva (2006) en el campo organizacional, con lo cual se puede observar la positiva acogida que está teniendo el ARS desde algunos años en este campo, ya que estos análisis, permiten identificar oportunidades de mejora que van a ayudar en el:

proceso de toma de decisiones y redes informales en una empresa (Molina, 2000), en la adopción de nuevas tecnologías (Burkhardt; Brass apud Zack, 2000) y en el impacto de los sistemas de mensajes electrónicos (Zack; Mckenney apud Zack, 2000), los cuales son relevantes para el área interdisciplinar constituida por la administración y por las $\mathrm{Cl}$ (2006, p.12, original en portugués).

\section{Interacciones documentales: un estudio de caso realizado desde el ARS para observar el comportamiento de diversas áreas de una entidad bancaria}

A comienzos del año 2013 se llevó a cabo un proyecto de Gestión Documental en una entidad financiera en la ciudad de Santiago de Cali, Colombia. Este proyecto debía realizarse para el cumplimiento de una ley que exigía a las entidades financieras realizar una metodología llamada Tablas de Retención Documental, donde se debía organizar los documentos físicos y electrónicos de las áreas que conformaban la empresa, establecer el tiempo de duración en archivos de gestión central e histórico, determinar su disposición final y su control de accesos. Este proyecto se realizó en ocho meses y se entrevistaron 173 personas para analizar el tipo de información que producían conforme a sus actividades inherentes a sus cargos.

De esta manera, este tipo de entrevistas se estructuraron en un formato llamado trámite donde se registraba toda la información que producía el encuestado: tiempo de labor del empleado en la entidad, las personas a las que le reportaba las actividades realizadas, el soporte de la información producida y las áreas a las que les compartía esa información (en caso de compartirla).

Con este recurso de información, se pretende realizar el ARS, ya que durante las entrevistas, el encuestado podía dar sus puntos de vista de novedades que él consideraba importantes para beneficio de la compañía, por lo que las encuestas no se usaron de una manera rígida y monotemática, sino que se ofreció una oportunidad al encuestado de expresarse de una forma libre.

Ahora, reutilizando esta información extraída del trámite de la información, se realizó una red social, donde no se incluyó todo el universo de personas entrevistadas en el proyecto de gestión documental, para efectos de una mayor facilidad de manejo de la red. La red se realizó con 72 actores, los cuales se estaban ubicados en 12 áreas administrativas: Auditoría, Gerencia Jurídica, Gerencia de Talento Humano, Gerencia de Operaciones, Gerencia de Tesorería, Unidad de Cumplimiento, Gerencia de Red, Front Office, Gerencia de Crédito, Gerencia de Mercadeo, Captaciones y Gerencia de Tecnología e Innovación.

En un primer momento se realizó una matriz asimétrica, es decir una tabla donde no todos los nodos se conectan unos con otros, "Si A refiere a B, no necesariamente B tiene que referir a A (Velázquez, Aguilar, 2005). La matriz fue realizada en Excel de forma cuadrada, donde los nodos que estaban en la fila, fueron los mismos que estaban en la columna y posteriormente se copió y pegó la tabla en el programa Ucinet como se observa en la siguiente imagen: 
Figura 6: Matriz asimétrica de los nodos y sus vínculos registrados en Ucinet.

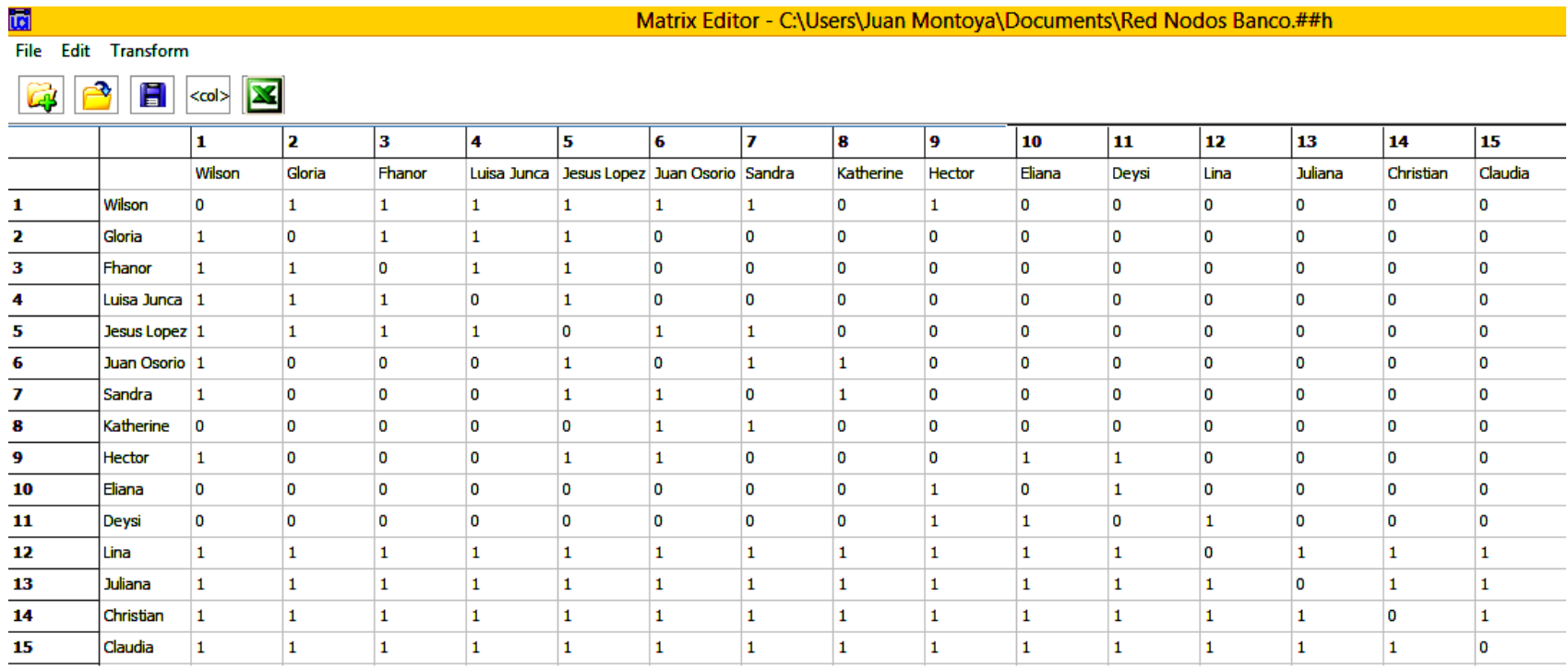

Fuente: Imagen de autoría propia

Los datos registrados en la anterior imagen establecen la relación de cada uno de los actores, dentro de la actividad documental en su día a día. Como se puede observar, el actor que tiene un vínculo 0 con otro actor no establece ningún tipo de relación documental, a diferencia del vínculo 1 donde se tiene un grado de relación. En esta matriz se puede ver que los actores que se cruzan con ellos mismo no tienen ningún grado de relación, por tanto se escribe 0 para no dejar la celda en blanco pues Ucinet no reconoce matrices que tienen espacios vacíos. Otro dato que es importante resaltar, es que se observó que al momento de estructurar la información, los nodos que estaban dentro de un área específica se conectaban entre sí, ya que la diseminación de la información antes de salir de cada una de las áreas, debía circular al interior, por tanto casi todos los nodos de las áreas en su interior, tienen un grado 1 de vínculo.

Posteriormente se utilizó la herramienta NetDraw para graficar la red y observar los vínculos de todos los nodos y las áreas al interior de la entidad bancaria. Se calculó inicialmente la densidad de la red para observar la alta o baja conectividad de los nodos al interior de la red: "La densidad es una medida expresada en porcentaje del cociente entre el número de relaciones existentes con las posibles" (Velázquez, Aguilar, 2005).

En este sentido, el porcentaje se genera de forma manual, calculando la red social del banco, la cual tiene 1631 relaciones de las 5512 posibles: se realizó multiplicando el número de nodos por su mismo número menos uno, es decir 72X71. Este valor se dividió y se multiplicó por 100, es decir 1631/5512X100, y el resultado obtenido fue la densidad de la red, que para este caso equivalió a $29.58 \%$.

Un atributo calculado en Ucinet en un primer momento, fue establecer el número de mujeres y hombres dentro de la red, donde se observó que hubo 36 mujeres y 36 hombres como se muestra a continuación: 
Figura 7: Red Social definidos entre los hombres y mujeres en cada una de las áreas administrativas

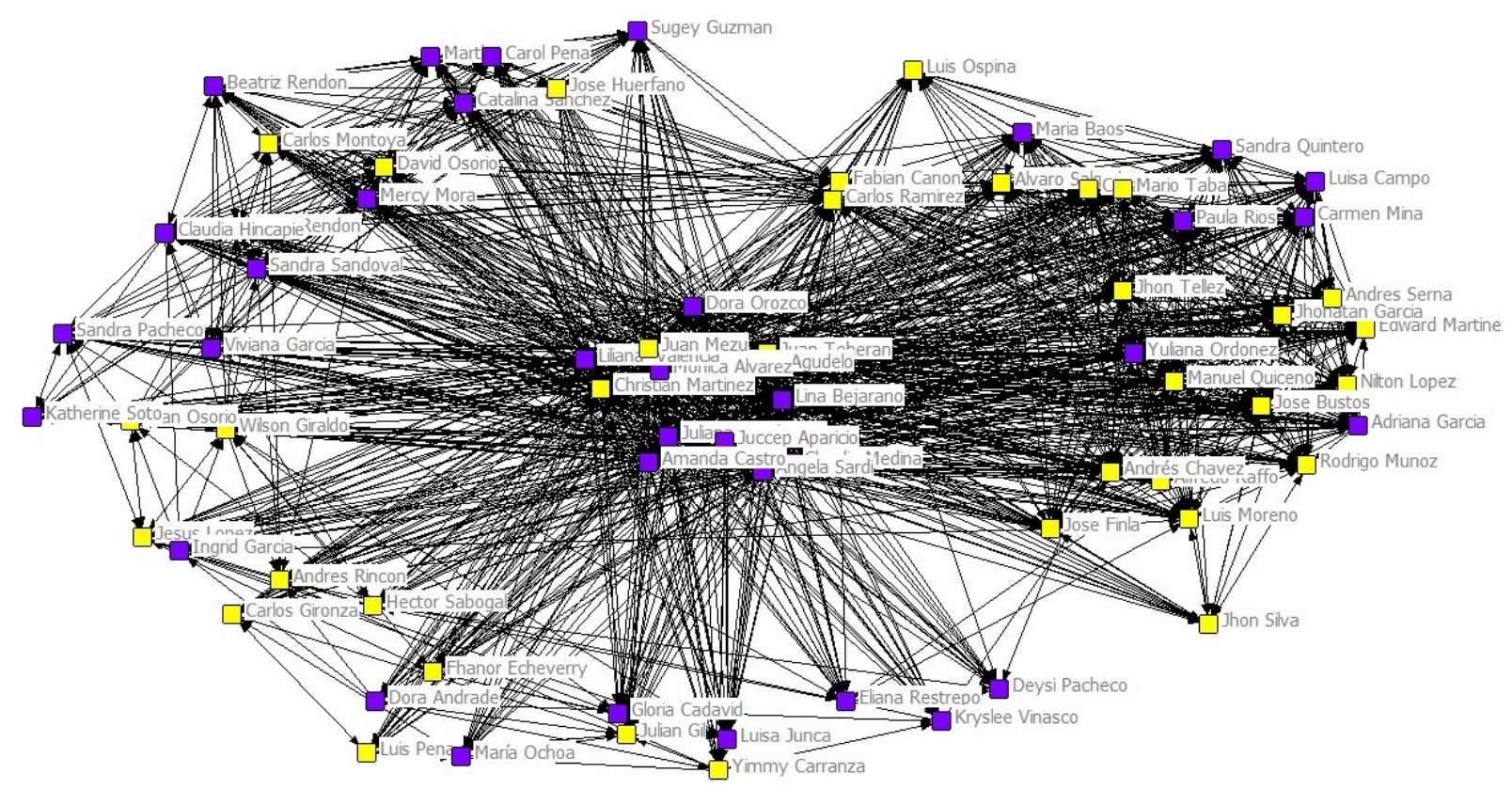

Fuente: Imagen de autoría propia

En un segundo momento, se realizó una gráfica donde se permitió ver con mayor claridad cada una de las áreas que componen la red social del banco. En esta gráfica se observa por ejemplo, el área de Gerencia de Talento Humano con un alto grado centralidad, debido a que es el departamento que establece una mayor relación con todos los nodos, no solo cuando un empleado ingresa por primera vez a la compañía sino a lo largo de toda su vida laboral, por tanto esta área es la que comparte información documental con el resto de las áreas de la red social:

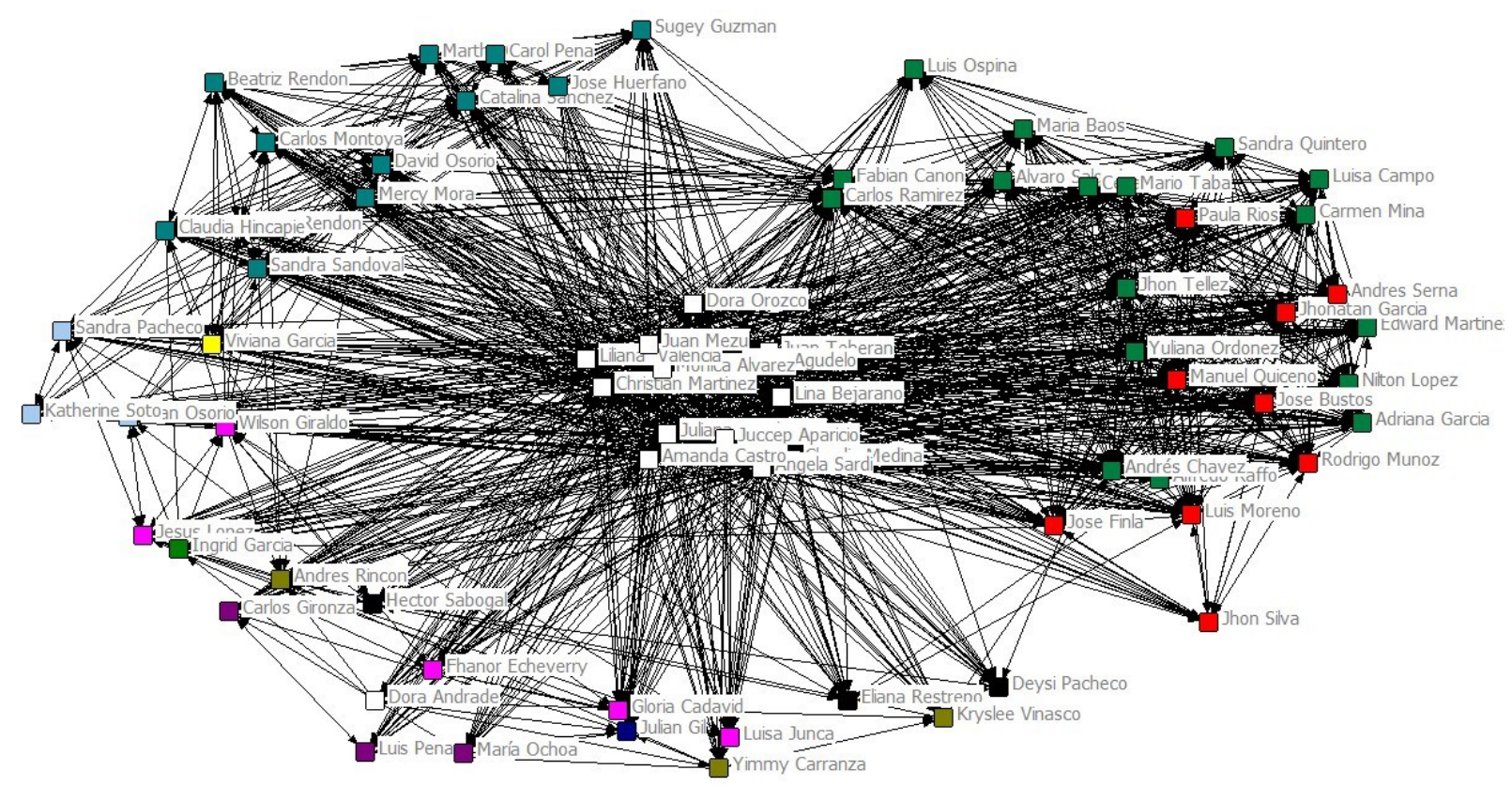

Fuente: Imagen de autoría propia 
Figura 8: Red por cada una de las áreas en la entidad bancaria - Continuación

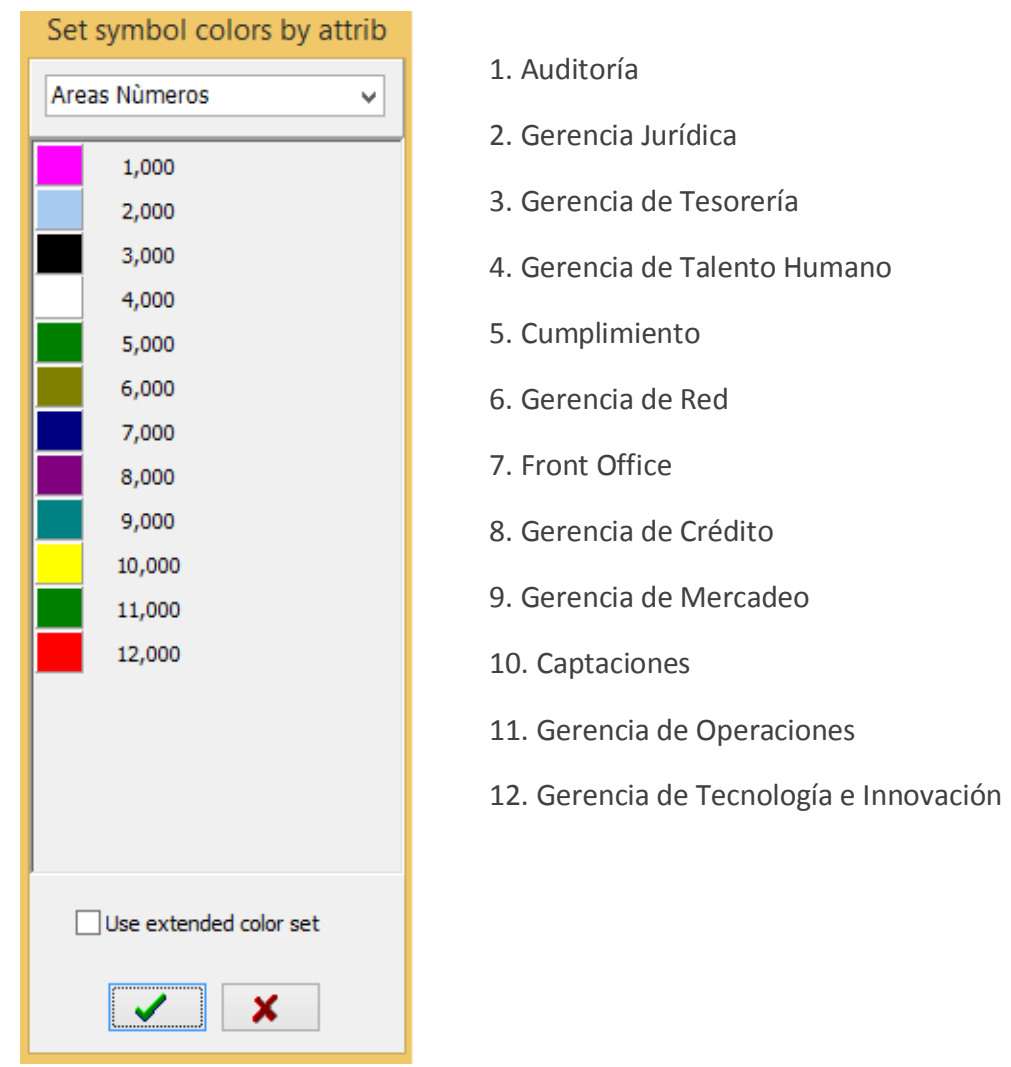

Fuente: Imagen de autoría propia

Seguidamente, se pasará a calcular en Ucinet, el Grado de Centralidad para establecer cuál es el nodo que está mayor conectado con los otros nodos en términos documentales:

Figura 9: Grado de Centralidad por nodo

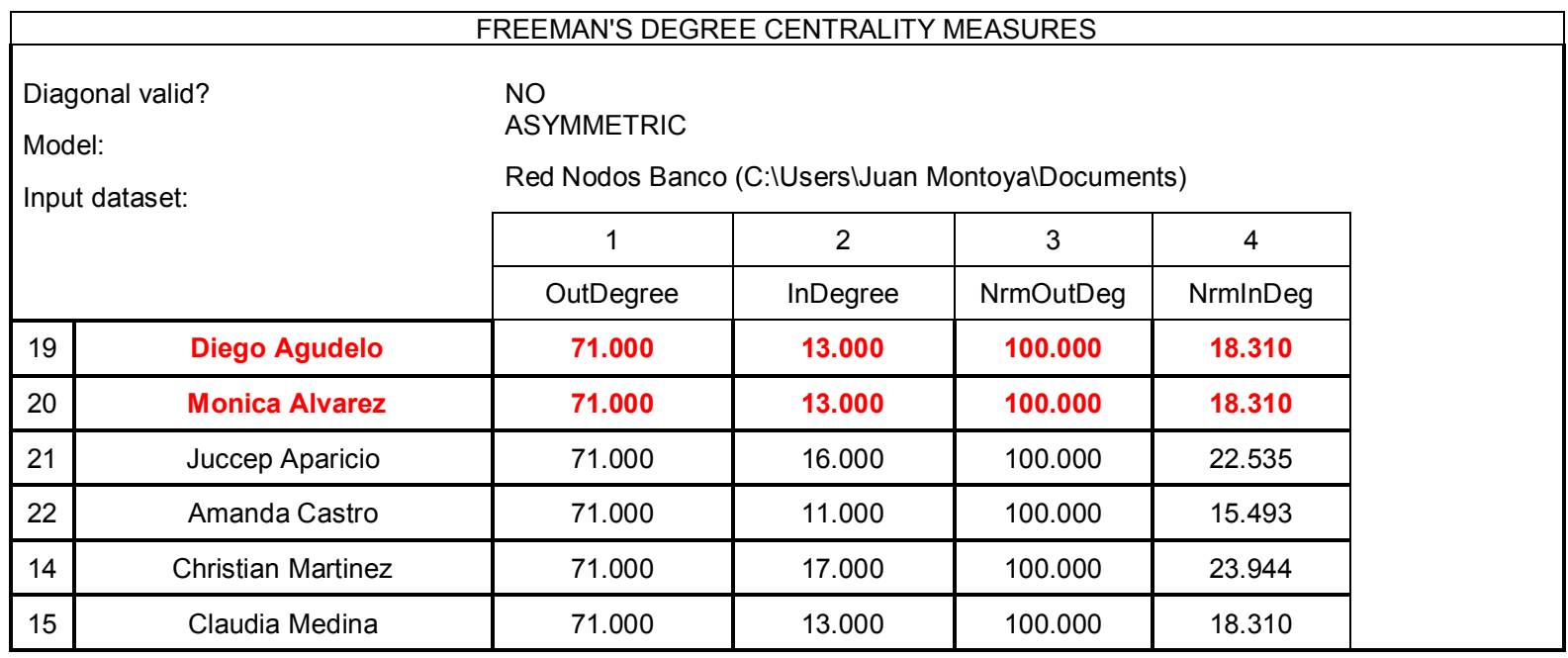


Figura 9: Grado de Centralidad por nodo - Continuación

\begin{tabular}{|c|c|c|c|c|c|}
\hline & & REEMAN'S DEG & CENTRALITY & EASURES & \\
\hline Diag & onal valid? & & & & \\
\hline Mod & & AS YIVIVIE IRIC & & & \\
\hline Innut & t dataset- & Red Nodos Ban & :IUsersIJuan & ntoyalDocume & \\
\hline & & 1 & 2 & 3 & 4 \\
\hline & & OutDegree & InDegree & NrmOutDeg & NrmlnDeg \\
\hline 16 & Angela Sardi & 71.000 & 13.000 & 100.000 & 18.310 \\
\hline 12 & Lina Bejarano & 71.000 & 14.000 & 100.000 & 19.718 \\
\hline 18 & Juan Teheran & 71.000 & 13.000 & 100.000 & 18.310 \\
\hline 13 & Juliana Panchanno & 71.000 & 13.000 & 100.000 & 18.310 \\
\hline 23 & Dora Orozco & 70.000 & 13.000 & 98.592 & 18.310 \\
\hline 25 & Juan Mezu & 70.000 & 13.000 & 98.592 & 18.310 \\
\hline 24 & Liliana Valencia & 70.000 & 13.000 & 98.592 & 18.310 \\
\hline 49 & Alfredo Raffo & 27.000 & 26.000 & 38.028 & 36.620 \\
\hline 48 & Carlos Ramirez & 27.000 & 38.000 & 38.028 & 53.521 \\
\hline 51 & Luisa Campo & 25.000 & 26.000 & 35.211 & 36.620 \\
\hline 47 & Fabian Canon & 25.000 & 39.000 & 35.211 & 54.930 \\
\hline 54 & Andres Chavez & 25.000 & 27.000 & 35.211 & 38.028 \\
\hline 58 & Maria Baos & 25.000 & 26.000 & 35.211 & 36.620 \\
\hline 52 & Adriana Garcia & 25.000 & 26.000 & 35.211 & 36.620 \\
\hline 61 & Yuliana Ordonez & 25.000 & 26.000 & 35.211 & 36.620 \\
\hline 62 & Cesar Astaiza & 24.000 & 26.000 & 33.803 & 36.620 \\
\hline 50 & Sandra Quintero & 24.000 & 26.000 & 33.803 & 36.620 \\
\hline 57 & Carmen Mina & 24.000 & 26.000 & 33.803 & 36.620 \\
\hline 53 & Alvaro Salgado & 24.000 & 27.000 & 33.803 & 38.028 \\
\hline 63 & Mario Taba & 24.000 & 26.000 & 33.803 & 36.620 \\
\hline 60 & Jhon Tellez & 24.000 & 26.000 & 33.803 & 36.620 \\
\hline & & DESCF & VE STATISTI & & \\
\hline & & 1 & 2 & 3 & 4 \\
\hline & & OutDegree & InDegree & NrmOutDeg & NrmlnDeg \\
\hline 1 & Mean & 22.653 & 22.653 & 31.905 & 31.905 \\
\hline 2 & Std Dev & 23.944 & 7.136 & 33.724 & 10.051 \\
\hline 3 & Sum & 1.631 .000 & 1.631 .000 & 2.297 .183 & 2.297 .183 \\
\hline 4 & Variance & 573.310 & 50.921 & 1.137 .294 & 101.014 \\
\hline 5 & SSQ & 78.225 .000 & 40.613 .000 & 155.177 .547 & 80.565 .367 \\
\hline 6 & MCSSQ & 41.278 .320 & 3.666 .319 & 81.885 .180 & 7.273 .000 \\
\hline 7 & Euc Norm & 279.687 & 201.527 & 393.926 & 283.840 \\
\hline 8 & Minimum & 0.000 & 11.000 & 0.000 & 15.493 \\
\hline 9 & Maximum & 71.000 & 39.000 & 100.000 & 54.930 \\
\hline 10 & $\mathrm{~N}$ of Obs & 72.000 & 72.000 & 72.000 & 72.000 \\
\hline Netu & vork Centralization $(\mathrm{O}$ & $.054 \%$ & & & \\
\hline Neth & vork Centralization (In & $49 \%$ & & & \\
\hline Acto & r-by-centrality matrix & et Red Nodos & $-\operatorname{deg}$ & & \\
\hline
\end{tabular}


Como se puede apreciar en la anterior figura, el área de talento humano, tuvo una mayor centralidad de grado debido al gran número de contactos con las otras áreas en términos documentales. Por otro lado, es importante también observar cómo los dos primeros nodos de esa misma área, tuvieron el mismo porcentaje de centralidad.

Esto es entendible porque en realidad Diego Agudelo, -el nodo con mayor centralidad de grado- es el auxiliar de nómina y compensación del área, lo que determina que la relación con sus pares sea esencial. Cuando se realizó la entrevista, el colaborador a pesar de tener un cargo poco estratégico para la entidad, el $100 \%$ de los empleados establecieron contactos documentales con este cargo, para su ingreso, desarrollo laboral y bienestar en la compañía.

Documentos como: créditos administrativos, auxilios de mantenimiento de vehículos, libranzas, convenios de bienestar con proveedores, memorandos de los créditos aprobados y actas de excepciones de créditos, resultaron tener un alto impacto y flujo tanto para todos los empleados como para la entidad financiera. Adicionalmente, el flujo documental no solo se dio al interior de las áreas de talento humano sino en varias áreas de la organización como planeación financiera, el comité de créditos (donde asisten gerentes estratégicos y tácticos de la empresa para aprobar o rechazar créditos), gerencia de operaciones, gerencia jurídica, entidades financieras externas y las agencias del propio banco que prestan el servicio dentro de la ciudad y en las otras sedes del país.

Por otro lado la gerente de talento humano Mónica Álvarez, tuvo el mismo porcentaje de vínculos. Por lo tanto su centralidad de grado es igualmente alta como se observa en la figura 9, ya que la interacción documental con la planta de empleados es la misma del auxiliar de nómina y compensación, ya que en este cargo se debe conocer todas las capacidades del talento humano que se está contratando y adicionalmente se debe hacer seguimiento a cada uno de los colaboradores que se contrata en el ámbito ocupacional y fuera de él.

Adicionalmente, la retroalimentación de cada una de las áreas que tiene a su cargo: bienestar y salud ocupacional, jefatura de selección, coordinación de relaciones laborales, nómina y compensación, jefatura de capacitaciones, coordinación de desarrollo, jefatura de comunicaciones y coordinación de contratación, es consolidado en un reporte de indicadores y presentado a la presidencia y a la gerencia general de la entidad financiera, con el objetivo de analizar el comportamiento organizacional periódicamente.

Finalmente, otro dato de relevancia que se observa en tabla anterior y en las gráficas 7 y 8 de NetDraw, es el fuerte grado de centralidad que tiene el área de talento humano en general, especialmente con sus primeros 13 nodos.

Ahora, a continuación se va a analizar la Centralidad de Intermediación, para observar el nodo que tiene el "control de comunicación" dentro de la red social del banco:

Figura 10: Centralidad de Intermediación por nodo

\begin{tabular}{|c|c|c|c|c|}
\hline \multicolumn{5}{|c|}{ FREEMAN BETWEENNESS CENTRALITY } \\
\hline $\begin{array}{l}\text { Input dataset: } \\
\text { Important note: }\end{array}$ & \multicolumn{4}{|c|}{$\begin{array}{l}\text { Red Nodos Banco (C: } \backslash \text { Users \Juan Montoya \Documents \Red Nodos Banco) } \\
\text { This routine cannot handle valued data, so it binarizes your data automatically. } \\
\text { It DOES handle directed (non-symmetric) data, so it does NOT symmetrize. }\end{array}$} \\
\hline \multicolumn{5}{|c|}{ Un-normalized centralization: 48276.630} \\
\hline & & 1 & 2 & \\
\hline & & Betweenness & nBetweenness & \\
\hline 1 & Wilson Giraldo & 753.148 & 15.154 & \\
\hline 21 & Juccep Aparicio & 593.090 & 11.933 & \\
\hline 47 & Fabian Canon & 514.358 & 10.349 & \\
\hline
\end{tabular}


Figura 10: Centralidad de Intermediación por nodo - Continuación

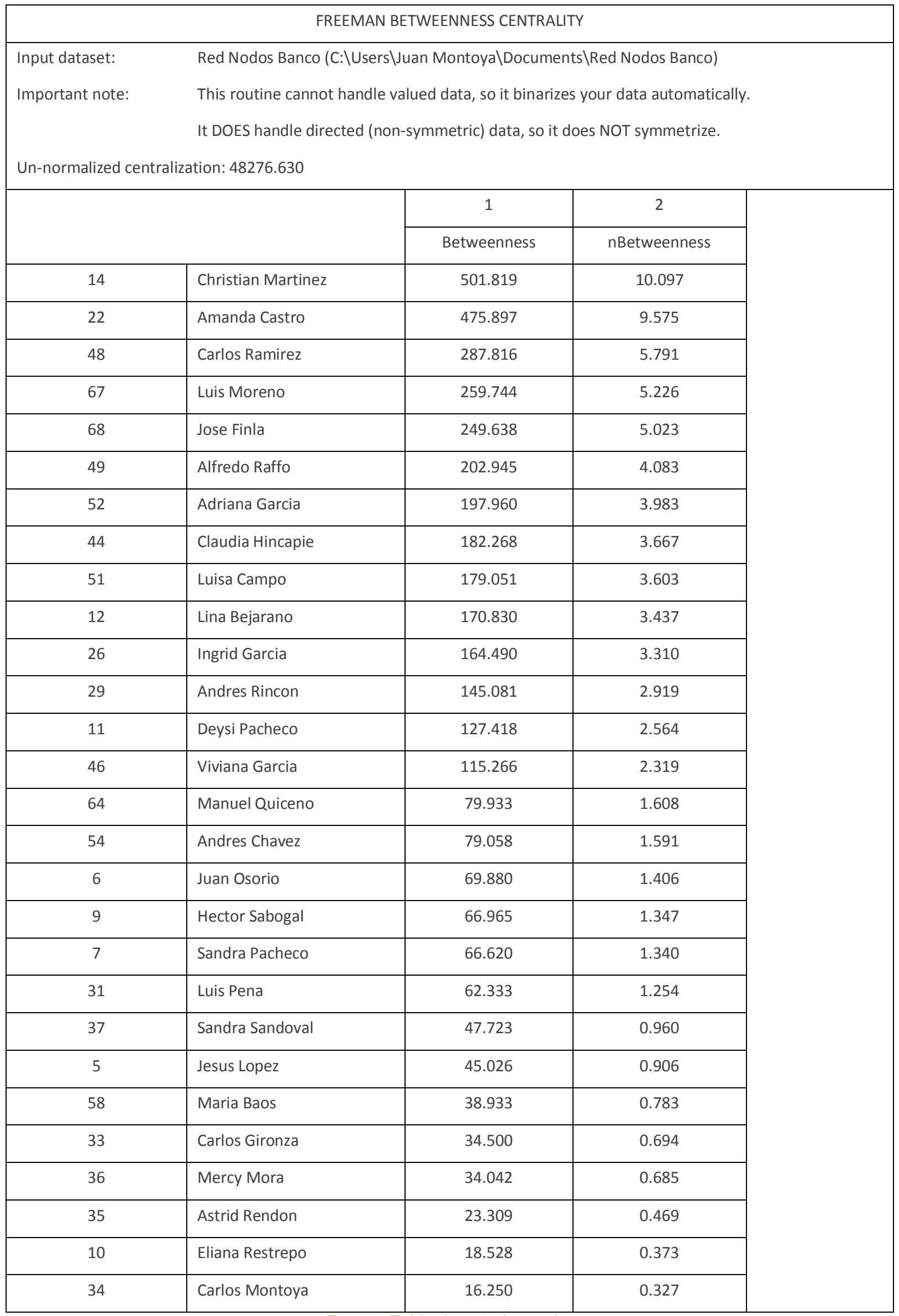


Figura 10: Centralidad de Intermediación por nodo - Continuación

\begin{tabular}{|c|c|c|c|}
\hline \multicolumn{4}{|c|}{ FREEMAN BETWEENNESS CENTRALITY } \\
\hline \multicolumn{4}{|c|}{ DESCRIPTIVE STATISTICS FOR EACH MEASURE } \\
\hline & & 1 & 2 \\
\hline & & Betweenness & nBetweenness \\
\hline 1 & Mean & 82.639 & 1.663 \\
\hline 2 & Std Dev & 152.578 & 3.070 \\
\hline 3 & Sum & 5.950 .000 & 119.718 \\
\hline 4 & Variance & 23.279 .938 & 9.425 \\
\hline 5 & SSQ & 2.167.857.000 & 877.643 \\
\hline 6 & MCSSQ & 1.676 .155 .500 & 678.581 \\
\hline 7 & Euc Norm & 1.472 .364 & 29.625 \\
\hline 8 & Minimum & 0.000 & 0.000 \\
\hline 9 & Maximum & 753.148 & 15.154 \\
\hline 10 & $\mathrm{~N}$ of Obs & 72.000 & 72.000 \\
\hline \multicolumn{4}{|c|}{ Network Centralization Index = 13.68\% } \\
\hline & neasure & ed Nodos Ba & \\
\hline
\end{tabular}

Fuente: Tabla de autoría propia.

(La tabla está resumida con el objetivo de analizar solo los principales nodos de la red)

Interpretando la imagen anterior generada por Ucinet, se observa que el actor que tiene la propiedad de conectar otros nodos es el Wilson Giraldo, el Auditor General de la empresa, con 753.148 grados, seguido por la analista de nómina de talento humano Juccep Aparicio con 593.090.

El auditor general de esta organización tiene una relación directa con cada una de las áreas y por ende con cada uno de los nodos dentro de ella, ya que las auditorías que se realizan diaria, semanal y mensualmente, le brindan la posibilidad de saber qué tipo de documentación se genera en cada uno de los departamentos, cuál es la importancia de estos documentos y cómo ellos circulan entre los actores de la entidad. En este sentido, si un nodo quiere entrar en contacto documental con otro nodo, la forma más rápida de hacerlo sería a través del auditor general.

Por último, el concepto nBetweenness que aparece en la última columna de la figura 10, indica el porcentaje normalizado en términos de grados, por tanto se puede observar que el grado normalizado de Wilson Giraldo es de $15.154 \%$, mientras que la analista de nómina, Juccep Giraldo, tiene un grado normalizado de $11.933 \%$.

Finalmente, no fue posible realizar el cálculo de Grado de Cercanía (Closenness) en Ucinet, ya que al momento de general el archivo en el programa, no se tuvo en cuenta que este tipo de cálculo solo se hace en matrices simétricas, es decir matrices donde los vínculos de los nodos son bidireccionales.

\section{Conclusiones}

Este trabajo permitió el análisis de una estructura organizacional desde un modelo alternativo, en el que se observó las relaciones de los autores dentro de un campo específico, permitiendo así con esta aproximación, dejar abierta la puerta para análisis más profundos y con diversos objetivos que puedan dar cuenta de las dificultades a las que se enfrenta una organización, especialmente cuando se trata del capital social al interior de ella. Por tanto, el ARS es una herramienta que puede ser aplicada dentro de las organizaciones para observar especialmente esos "cuellos de botella" que dificultan las interacciones entre los colaboradores y no permiten el avance deseado por la compañía. 
Este estudio permitió observar que la entidad bancaria tiene un área consolidada como la Gerencia de Talento Humano, la cual establece un contacto documental con las demás áreas. Sin embargo, se observó igualmente que Gerencias como Operaciones, Tesorería, Red y Jurídica, no tienen vínculos importantes entre sus actores, por lo que se podría generar un plan de acción para establecer un diálogo con ellos, o reestructurar los colaboradores de estas áreas, en lugares más acordes con su rendimiento laboral.

Igualmente, se observó por ejemplo que de los tres actores de la Gerencia de Tesorería, dos establecen vínculos fuertes con las demás áreas, a diferencia del Gerente del área, el cual no tiene un papel preponderante dentro y fuera del área. Igualmente, dentro del área de Gerencia de Operaciones, hay un actor que no establece un vínculo documental con ningún otro actor, por lo que es importante "ir al terreno" laboral para analizar la importancia de este actor dentro del área.

Finalmente, sería interesante encontrar en estudios futuros por medio del ARS en organizaciones, no solo la información documental en soporte papel que los actores producen y tramitan fruto de sus funciones, sino, aquella información que se encuentra en otro tipo de soportes como lo son por ejemplo, la información digital que se encuentran en los sistemas de gestión, en los servidores de las compañías, en nubes externas o internas y en los computadores personales de los empleados. Así mismo, analizar a profundidad la totalidad de los colaboradores que producen y gestionan la información de la empresa en un archivo de gestión, en un archivo central y hasta en un archivo histórico.

La realización de este trabajo se dio principalmente por las herramientas investigativas que en la actualidad ofrece el ARS, donde como se observó a lo largo del texto, fue aplicada a diversos dominios teóricos y prácticos y en la mayoría de los casos. Los resultados generados fueron totalmente diferentes a los que se realizaban por vías tradicionales. Ahora bien, el Análisis de Redes Sociales aplicado a las organizaciones y especialmente al ámbito documental, resalta no sólo la importancia del flujo documental, si no también, a los protagonistas que producen y tramitan esos documentos, proponiendo alternativas para observar una entidad en su "estado puro", analizando el comportamiento de los actores en cada una de las áreas donde se desenvuelven y dónde ellos pueden ser más productivos, elemento preponderante tanto para el beneficio del propio actor como para la entidad que lo contrata.

Por último como se pudo observar, la utilización del ARS dentro de las organizaciones tiene más ventajas que desventajas, y su éxito dependerá del objetivo que se quiera determinar al momento de estudiar la red social.

\section{Bibliografía}

Fialho-Joaquim, Rita-Moreno. (2015). O trabalho no setor bancário. Um estudo sobre a cooperação interorganizacional numa rede de agências bancárias. REDES- Revista hispana para el análisis de redes sociales, v.26, n.1, pp.149-166. http://dx.doi.org/10.5565/rev/redes.545

García-Macías, Alejandro. (2002). Redes sociales y clusters empresariales. REDES, Revista Hispana para el Análisis de Redes Sociales, v.1, n.6, pp.1-20. http://revista-redes.rediris.es/html-vol1/vol1 6.htm

Marqués-Sánchez, Pilar; González-Pérez, Marta-Eva; Agra-Varela, Yolanda; Vega-Núñez, Jorge; Pinto-Carral, Arrate; Quiroga-Sanchéz, Enedina. (2013). El análisis de redes sociales. Un método para la mejora de la seguridad en las organizaciones sanitarias. Revista Española de Salud Pública, v.87, n.3, pp.210-219.

http://scielo.isciii.es/scielo.php?pid=S1135-57272013000300001\&script=sci arttext

Marteleto, Regina-María. (2001). Analise de redes sociais-Aplicação nos estudos de transferência da informação. Ciência da Informação, v.30, n.1, pp.71-81 http://repositorio.ibict.br/handle/123456789/187

Matheus, Renato-Fabiano; Silva-De-Oliveira, Antonio. (2006). Análise de redes sociais como metodo para a Ciência da Informação. DataGramaZero, Revista de Ciência da Informação, v.7 n.2, pp.1-26. http://www.dgz.org.br/abr06/Art 03.htm

Molina-González, José-Luís. (2005). El estudio de las redes personales: contribuciones, métodos y perspectivas. EMPIRIARevista de Metodología de Ciencias Sociales, n.10, pp.71-106. doi:http://dx.doi.org/10.5944/empiria.10.2005

Otte, Evelien; Rousseau, Ronald. (2002). Social network analysis: a powerful strategy, also for the information sciences. Journal de Information Science, v.28, n.6, pp.441-453.

http://www.elsevier.com/search?query=Social+network+analysis $\% 3 \mathrm{~A}+\mathrm{a}+$ powerful+strategy $\% 2 \mathrm{C}+$ also+for+the+information+sci $\underline{\text { ences }}$

Pina-Stranger, Álvaro. (2012). Introducción: el análisis de redes inter-organizacionales. REDES, Revista hispana para el análisis de redes sociales, v.23, n.1, pp.1-6. http://revistes.uab.cat/redes/article/view/436 
Rodríguez-Treviño, Júlio-César. (2013). Cómo utilizar el análisis de redes sociales para temas de historia. Signos Históricos, n.29, pp.102-141. http://dcsh.izt.uam.mx/publicaciones/filosofia/index.php/SH/index

Velásquez-Álvarez, Alejandro; Aguilar-Gallegos, Norman. (2005). Manual introductorio al análisis de redes sociales. REDES, Revista Hispana de Análisis de Redes Sociales, pp.1-45. http://revista-redes.rediris.es/html-vol1/vol1_6.htm

Wasserman, Stanley; Faust, Katherine. (1997). Social network analysis: methods and applications. New York, Estados Unidos: Cambridge Univesity Press.

\section{Datos de los autores}

Juan Bernardo Montoya Mogollón

Profesional formado en historia de la Universidad del Valle, con experiencia en elaboración y aplicación de tablas de retención documental en empresas colombianas. Maestrante de Ciencias de la Información en la Universidade Estadual Paulista Júlio de Mesquita Filho - UNESP.

juanmont1981@gmail.com

\section{Telma Campanha de Carvalho Madio}

Posee graduación en Historia por la Pontifícia Universidade Católica de São Paulo (1985), especialización en Archivos por el IEB/USP (1988), máster en Historia por la Pontifícia Universidade Católica de São Paulo (1999) y doctorado en Ciencias de la Comunicación por la Universidade de São Paulo (2005). Actualmente es profesora asistente de la Universidade Estadual Paulista Júlio de Mesquita Filho - UNESP, en el Departamento de Ciência da Informação de la Faculdade de Filosofia e Ciências - Campus Marília, administrando disciplinas en la graduación y en el posgrado. Coordinadora del Laboratório de Conservação, desde 2006. En diversos periodos coordinadora del curso de Arquivologia y actualmente miembro titular del Conselho de Curso e Departamental. Miembro de la Comissão de Avaliação Documental e Acesso/CADA de la UNESP. Actua en las líneas de investigación producción, organización de la información y gestión de la información, con los temas: organización e identificación archivística, fotografía, acervos audiovisuales, conservación preventiva e Historia Contemporánea de Brasil.

telmaccarvalho@marilia.unesp.br

Recibido - Received: 2016-01-13

Aceptado - Accepted: 2016-03-19

\section{$(\mathrm{cc}) \mathrm{Br}$}

This work is licensed under a Creative Commons Attribution 4.0 United States License.

\section{UILIS D.}

This journal is published by the University Library System of the University of Pittsburgh as part of its $\underline{D-S c r i b e}$ Digital Publishing Program and is cosponsored by the University of Pittsburgh Press. 